\title{
A GENERALIZATION OF THE COFINITENESS PROBLEM IN LOCAL COHOMOLOGY MODULES
}

\author{
J. ASADOLLAHI, K. KHASHYARMANESH and SH. SALARIAN
}

(Received 3 February 2001; revised 25 September 2002)

Communicated by J. Du

\begin{abstract}
Let $R$ be a commutative Noetherian ring with nonzero identity and let $M$ be a finitely generated $R$ module. In this paper, we prove that if an ideal $I$ of $R$ is generated by a u.s.d-sequence on $M$ then the local cohomology module $H_{i}^{i}(M)$ is $I$-cofinite. Furthermore, for any system of ideals $\Phi$ of $R$, we study the cofiniteness problem in the context of general local cohomology modules.
\end{abstract}

2000 Mathematics subject classification: primary 13D03, $13 \mathrm{D} 45$.

Keywords and phrases: Local cohomology, cofinite, $d$-sequence, Bass number.

\section{Introduction}

Throughout this paper, $R$ denotes a commutative Noetherian ring with non-zero identity and $M$ is a finitely generated $R$-module. We shall only assume that $R$ is local, when this is explicitly stated.

For a Noetherian local ring $R$ with the maximal ideal $\mathfrak{m}$ and a finitely generated $R$-module $M$, it is well known that the local cohomology modules $H_{\mathrm{m}}^{j}(M)$ are Artinian. It is an easy consequence of Matlis duality [21] and the basic work of Grothendieck [10] that this property is equivalent to say that $\operatorname{Supp}_{R}\left(H_{\mathrm{m}}^{j}(M)\right) \subseteq V(\mathfrak{m})$ and $\operatorname{Hom}_{R}\left(k, H_{\mathrm{m}}^{j}(M)\right)$ is finitely generated. In view of these facts, the following conjecture w'as made by Grothendieck (see [11, Expose XIII, Conjecture 1.2]):

CONJECTURE 1.1. Let $M$ be a finitely generated $R$-module and let $I$ be an ideal of $R$. Then the module $\operatorname{Hom}_{R}\left(R / I, H_{I}^{j}(M)\right)$ is finitely generated for all $j \geq 0$.

(C) 2003 Australian Mathematical Society $1446-7887 / 03 \$ A 2.00+0.00$ 
The conjecture is false in general, as was shown by Hartshorne [12] with a counterexample, which states that the conjecture is not true even if the ring $R$ is regular. Hartshorne defined a module $N$ to be $I$-cofinite if the support of $N$ is contained in $V(I)$ and $\operatorname{Ext}_{R}^{i}(R / I, N)$ is finitely generated for all $i$ and asked the following question:

QUESTION 1.2. If $l$ is an ideal of $R$ and $M$ is a finitely generated $R$-module, when are $\operatorname{Ext}_{R}^{i}\left(R / I, H_{l}^{j}(M)\right)$ finitely generated for all $i$ and all $j$ ?

By working in the derived category, Hartshorne showed that if $M$ is a finitely generated $R$-module, where $R$ is a complete regular local ring, then $H_{I}^{j}(M)$ is $I$ cofinite in two cases:

(i) $I$ is a non-zero principal ideal [12, Corollary 6.3].

(ii) $I$ is a prime ideal with dimension 1 [12, Corollary 7.7].

There are several papers devoted to the extension of Hartshorne's second result to more general situations. We refer the reader to the papers of Huneke and Koh [14, Theorem 4.1], Delfino [8, Theorem 3], Delfino and Marley [9, Theorem 1], and Yoshida [29, Theorem 1.1].

On the other hand, there have been attempts to generalize result (i) for a ring without any restriction. The general case was proved by Kawasaki [17]. He showed that if an ideal $I$ of a Noetherian ring is principal, up to radical, then the local cohomology modules with support in $V(I)$ are $I$-cofinite.

There are some generalizations of the theory of local cohomology modules. The following is given in [3].

DEFINITION 1.1. Let $\Phi$ be a non-empty set of ideals of $R$. We call $\Phi$ a system of ideals (of $R$ ) if, whenever $I, I^{\prime} \in \Phi$, then there is an ideal $J \in \Phi$ such that $J \subseteq I I^{\prime}$. For such a system, for every $R$-module $M$, one can define

$$
\Gamma_{\Phi}(M)=\{x \in M: J x=0 \text { for some } J \in \Phi\} .
$$

Then $\Gamma_{\Phi}(-)$ is a functor from $\mathscr{C}(\mathscr{R})$ to itself (where $\mathscr{C}(\mathscr{R})$ denotes the category of all $R$-modules and all $R$-homomorphisms).

The functor $\Gamma_{\Phi}(-)$ is additive, covariant, $R$-linear and left exact. In [3], $\Gamma_{\Phi}(-)$ is denoted by $L_{\Phi}(-)$ and is called the 'general local cohomology functor with respect to $\Phi$ '. For each $i \geq 0$, the $i$-th right derived functor of $\Gamma_{\Phi}(-)$ is denoted by $H_{\Phi}^{i}(-)$. It is shown that the study of torsion theory over $R$ is equivalent to study the general local cohomology theory (see [3]).

The purpose of this note is to make a suitable generalization of Conjecture 1.1 and Question 1.2 in the context of general local cohomology modules and then obtain some results as above, especially for Hartshorne's first result, but for general local cohomology modules. 
To go into a detail about our main results, let us begin with a definition.

DEFINITION 1.2. Let $\Phi$ be a system of ideals of $R$. The general local cohomology module $H_{\Phi}^{j}(M)$ is defined to be $\Phi$-cofinite if there exists an ideal $I \in \Phi$ such that $\operatorname{Ext}_{R}^{i}\left(R / I, H_{\Phi}^{j}(M)\right)$ is finitely generated for all $i$.

Concerning this, we are led to the following analogue of Conjecture 1.1 and Question 1.2 in the general local cohomology context:

QUESTION 1.3. Let $\Phi$ be a system of ideals of $R$ and $I \in \Phi$. Let $M$ be a finitely generated $R$-module. When is $\operatorname{Hom}_{R}\left(R / I, H_{\Phi}^{j}(M)\right)$ finitely generated?

QUESTION 1.4. Let $\Phi$ be a system of ideals of $R$. Let $M$ be a finitely generated $R$-module. When are $H_{\Phi}^{j}(M) \Phi$-cofinite for all $j$ ?

The origins of our idea go back to the following lemma of Huneke and Koh:

LEMMA 1.3 ([14, Lemma 4.2]). Let $I$ be an ideal of a Noetherian ring $R$. Then $H_{I}^{j}(M)$ is $I$-cofinite if and only if $H_{I}^{j}(M)$ is $I^{\prime}$-cofinite, where $I^{\prime}$ is the radical of $I$.

This result tells us that, in one special case at least, where $\Phi=\left\{I^{i}: i \geq 1\right\}$, our generalization is compatible with the original ones.

However, after some preliminary results in section one, we provide partial answers to the above questions. For instance, we prove that whenever $\Phi$ is a system of ideals of $R, M$ is a finitely generated $R$-module and $n$ is a positive integer such that $H_{\Phi}^{j}(M)$ is finitely generated for all $j<n$ and that $H_{J}^{j}(M)=0$ for all $j>n$ and all $J \in \Phi$, then, $H_{\Phi}^{i}(M)$ is $\Phi$-cofinite for all $i$. We also show that if $n$ is a non-negative integer such that for an ideal $l$ of a Noetherian ring $R$ and a finitely generated $R$-module $M, H_{I}^{i}(M)$ is finitely generated for all $i<n$, then $\operatorname{Hom}_{R}\left(R / I, H_{I}^{n}(M)\right)$ is finitely generated. By the same argument, we will be able to extend the result to the context of general local cohomology modules.

\section{Preliminaries}

In this section we review some definitions and results. We begin with the following definition

DEFINITION 2.1. A sequence $x_{1}, \ldots, x_{n}$ of elements of $I$ is said to be an $I$-filter regular $M$-sequence, if $\operatorname{Supp}_{R}\left(\left(x_{1}, \ldots, x_{i-1}\right) M:_{M} x_{i} /\left(x_{1}, \ldots, x_{i-1}\right) M\right) \subseteq V(I)$ for all $i=1, \ldots, n$, where $V(I)$ denotes the set of prime ideals of $R$ containing $I$. 
This concept is a generalization of the one of a filter regular sequence which has been studied in $[25,27,28]$ and has led to some interesting results. Both concepts coincide if $I$ is the maximal ideal of a local ring. It is easy to see that the analogue of [27, Appendix 2 (ii)] holds true whenever $M$ is finitely generated and $\mathfrak{m}$ replaced by $I$; so that, if $x_{1}, \ldots, x_{n}$ is an $I$-filter regular $M$-sequence, then there is an element $y \in I$ such that $x_{1}, \ldots, x_{n}, y$ is an $I$-filter regular $M$-sequence. Thus, for a positive integer $n$, there exists an $I$-filter regular $M$-sequence of length $n$.

Throughout the paper, we shall appeal to the following proposition without further comments.

PROPOSITION 2.2 (Nagel-Schenzel). Let $x_{1}, \ldots, x_{n} \in I$ be an I-filter regular $M$ sequence. Then there are the following isomorphisms $H_{l}^{i}(M) \cong H_{\left(x_{1}, \ldots, x_{n}\right)}^{i}(M)$ for all $i<n$, and $H_{I}^{i}(M) \cong H_{l}^{i-n}\left(H_{\left(x_{1}, \ldots, x_{n}\right)}^{n}(M)\right)$ for all $i \geq n$.

Proof. See [22, Lemma 3.4] and [18, Proposition 1.2].

DEFINITION 2.3. A sequence $x_{1}, \ldots, x_{n}$ of elements of $R$ is said to be an $I$-weak $M$-sequence if $\left(x_{1}, \ldots, x_{i-1}\right) M:_{M} x_{i} \subseteq\left(x_{1}, \ldots, x_{i-1}\right) M:_{M} I$ for all $i=0, \ldots, n$.

REMARK 2.4. Clearly every $I$-weak $M$-sequence in $I$ is an $I$-filter regular $M$ sequence.

PROPOSITION 2.5. For a system of ideals $\Phi$ of a Noetherian ring $R$, a finitely generated $R$-module $M$, and a positive integer $n$, the following conditions are equivalent:

(1) $H_{\Phi}^{j}(M)$ is finitely generated for all $j<n$.

(2) There exists an ideal $I$ in $\Phi$ such that $I H_{\Phi}^{j}(M)=0$ for all $j<n$.

(3) There exists an ideal $I$ in $\Phi$ such that, for all $J \in \Phi$, every $J$-filter regular $M$-sequence, of length $n$, is an I-weak $M$-sequence.

Proof. See [1, Theorem 3].

For convenience we write $H_{\Phi}^{j}(M)=0$, whenever $j$ is a negative integer. Also, for an ideal $I$ of $R$, we use $D_{l}(-)$ to denote the functor $\lim _{\bar{\alpha}} \operatorname{Hom}_{R}\left(I^{\alpha},-\right)$.

\section{Cofiniteness of local cohomology modules for an ideal generated by a u.s. $d$-sequence}

DEFINITION 3.1. The sequence $x_{1}, \ldots, x_{n}$ of elements of $R$ is called a $d$-sequence on $M$ if, for each $i=0,1, \ldots, n-1$, the equality

$$
\left(x_{1}, \ldots, x_{i}\right) M:_{M} x_{i+1} x_{k}=\left(x_{1}, \ldots, x_{i}\right) M:_{M} x_{k}
$$


holds for all $k \geq i+1$ (this is actually slight weakening of Huneke's definition in [13]); it is an unconditioned strong $d$-sequence (a u.s. $d$-sequence) on $M$ if $x_{1}^{\alpha_{1}}, \ldots, x_{n}^{\alpha_{n}}$ is a $d$-sequence in any order for all positive integers $\alpha_{1}, \ldots, \alpha_{n}$.

REMARK 3.2. If $I$ is an ideal generated by a u.s. $d$-sequence $x_{1}, \ldots, x_{n}$ on $M$, then $0:_{M} x_{i}^{l}=0:_{M} x_{i}=0:_{M} x_{i} x_{j}=0:_{M} x_{j}$ for all $i, j$ with $1 \leq i, j \leq n$ and all $l \geq 1$ and so $\Gamma_{l}(M)=\left(0:_{M} x_{i}\right)$ for all $i=1,2, \ldots, n$.

REMARK 3.3. If $I$ is an ideal generated by a u.s. $d$-sequence $x_{1}, \ldots, x_{n}$ on $M$, then $x_{1}, \ldots, x_{n}$ is an $I$-filter regular $M$-sequence.

THEOREM 3.4. If I is an ideal generated by a u.s.d-sequence $x_{1}, \ldots, x_{n}$ on $M$, then the local cohomology module $H_{I}^{i}(M)$ is I-cofinite for all $i$.

REMARK 3.5. Note that, by [29, Proposition 3.1], in the above theorem $H_{I}^{n}(M)$ is not finitely generated, unless $H_{I}^{i}(M)=0$ for all $i>0$.

PROOF OF THEOREM 3.4. It is well known that $H_{I}^{i}(M)=0$ for all $i>n$. Also, by Remark 3.3 and [19, Theorem A], $H_{I}^{i}(M)$ is finitely generated for all $i<n$. Hence we need only to show that $\operatorname{Ext}_{R}^{j}\left(R / I, H_{I}^{n}(M)\right)$ is finitely generated for all $j \geq 0$. To do this, we use induction on $n$, the length of the sequence. When $n=0$, there is nothing to prove. Assume, inductively, that $n \geq 1$ and that the result has been proved for positive integer smaller than $n$. Since $x_{1}, \ldots, x_{n}$ is a u.s.d-sequence on $M$, by Remark 3.2, $H_{I}^{0}(M)=\left(0:_{M} x_{1}\right)$. Now, in view of the natural isomorphism $H_{I}^{n}\left(M /\left(0:_{M} x_{1}\right)\right) \cong H_{I}^{n}(M)$, we may replace $M$ by $M /\left(0:_{M} x_{1}\right)$ and assume that $x_{1}$ is a non-zero divisor on $M$. Set $\bar{M}:=M / x_{1} M$ and $J:=\left(x_{2}, \ldots, x_{n}\right)$. It is clear that $x_{2}, \ldots, x_{n}$ is a u.s. $d$-sequence on $\bar{M}$ and so, by the inductive hypothesis, $H_{J}^{n-1}(\bar{M})$ is $J$-cofinite. Since, by the Independence Theorem, $H_{I}^{n-1}(\bar{M}) \cong H_{J}^{n-1}(\bar{M})$, it follows from [14, Lemma 4.2] that $H_{l}^{n-1}(\bar{M})$ is $I$-cofinite. Consider the exact sequence

$$
0 \rightarrow M \stackrel{x_{1}}{\rightarrow} M \rightarrow \bar{M} \rightarrow 0
$$

to obtain the long exact sequence

$$
H_{I}^{n-1}(M) \stackrel{f}{\rightarrow} H_{I}^{n-1}(\bar{M}) \stackrel{g}{\rightarrow} H_{I}^{n}(M) \stackrel{x_{1}}{\rightarrow} H_{I}^{n}(M) \rightarrow 0 .
$$

By $\left[19\right.$, Theorem A], $H_{I}^{n-1}(M)$ is finitely generated and so is $\operatorname{Im} f$. Therefore we conclude from the exact sequence $0 \rightarrow \operatorname{Im} f \rightarrow H_{I}^{n-1}(\bar{M}) \rightarrow \operatorname{Im} g \rightarrow 0$ that $\operatorname{Im} g$ is $I$-cofinite. Now, using the exact sequence

$$
0 \rightarrow \operatorname{Im} g \rightarrow H_{I}^{n}(M) \stackrel{x_{1}}{\rightarrow} H_{I}^{n}(M) \rightarrow 0
$$

and the long exact sequence of 'Ext' modules, by noting that $x \in I$, we obtain that $\operatorname{Ext}_{R}^{j}\left(R / I, H_{I}^{n}(M)\right)$ is finitely generated as required. 
The next theorem is another main result of this paper.

THEOREM 3.6. Let $M$ be a finitely generated $R$-module and $I$ be an ideal of $R$. Suppose that $n \geq 0$ is such that $H_{I}^{j}(M)$ is finitely generated for all $j<n$ and that $H_{l}^{j}(M)=0$ for all $j>n$. Then $H_{l}^{i}(M)$ is $I$-cofinite for all $i$.

ProOF. We only need to prove the result for $i=n$. By [19, Theorem A], there exists a u.s. $d$-sequence $x_{1}, \ldots, x_{n}$ on $M$ which is an $I$-filter regular $M$-sequence. Put $N:=H_{\left(x_{1}, \ldots, x_{n}\right)}^{n}(M)$. Hence $H_{I}^{0}(N) \cong H_{I}^{n}(M)$ and $H_{l}^{l}(N) \cong H_{I}^{n+1}(M)=0$. Also, by [7, Theorem 2.2 .4$]$, we have the exact sequence

$$
0 \rightarrow H_{I}^{n}(M) \rightarrow N \rightarrow D_{l}(N) \rightarrow 0 .
$$

Now, using the following claim in conjunction with [14, Lemma 4.2], we may assume that $I$ can be generated by a u.s. $d$-sequence on $M$, of length $n$. Now the result follows from Theorem 3.4.

CLAIM 3.7. Let $M$ and $I$ be as above and let $x_{1}, \ldots, x_{n}$ be a u.s.d-sequence on $M$ which is an I-filter regular $M$-sequence. Put $N:=H_{\left(x_{1}, \ldots, x_{n}\right)}^{n}(M)$. Then

$$
\operatorname{Ext}_{R}^{i}\left(R / I, D_{I}(N)\right)=0
$$

for all $i \geq 0$.

Progr, Let $E$ be an iniective $R$-module. Then by 7 , Theorem 2.24 (ill, the sequence

$$
0 \rightarrow \Gamma_{I}(E) \rightarrow E \rightarrow D_{I}(E) \rightarrow 0
$$

is exact. Since, by [7, Proposition 2.1.4], $\Gamma_{I}(E)$ is injective, the above sequence is split and so $D_{l}(E)$ is injective. Now, let $L^{\bullet}$ be an injective resolution of $N$. Thus $D_{l}\left(L^{\bullet}\right)$ is an injective complex, whose terms, by [7, Corollary 2.2 .8 (iv)], are all $I$-torsion-free. Note that, by [7, Theorem 2.2 .4 (ii)], $\mathscr{R}^{i} D_{l}(N) \cong H_{I}^{i+1}(N)=0$ for all $i \geq 1$ and $D_{l}(-)$ is a left exact functor. Hence $D_{l}\left(L^{\bullet}\right)$ is an injective resolution of $D_{I}(N)$ and so $\operatorname{Ext}_{R}^{i}\left(R / I, D_{I}(N)\right)=0$ for all $i \geq 0$.

\section{Results on cofiniteness of general local cohomology modules}

The following theorem is one of the main results of this paper. With the following theorem, we not only offer a partial answer to the Question 1.3, but we also provide an improved form of Proposition 6 of [1] (see [11, Expose XIII, Conjecture 1.1]). 
THEOREM 4.1. Let $I$ be an ideal of a Noetherian ring $R$ and $M$ be a finitely generated $R$-module. Let $n$ be a non-negative integer such that $H_{I}^{i}(M)$ is finitely generated for all $i<n$. Then $\operatorname{Hom}_{R}\left(R / I, H_{l}^{n}(M)\right)$ is finitely generated.

PROOF. We use induction on $n$. When $n=0$, the module $H_{l}^{0}(M) \cong \Gamma_{l}(M)$ is finitely generated. Assume, inductively, that $n>0$ and we have established the result for positive integers smaller than $n$. Consider the exact sequence

$$
0 \rightarrow \Gamma_{I}(M) \rightarrow M \rightarrow \frac{M}{\Gamma_{I}(M)} \rightarrow 0 .
$$

By [7, Corollary 2.1 .7 (ii)], $H_{l}^{i}\left(\Gamma_{I}(M)\right)=0$ for all $i>0$. This means that $H_{l}^{i}(M) \cong$ $H_{I}^{i}\left(M / \Gamma_{I}(M)\right)$, for all $i>0$. Hence we can make the additional assumption that $M$ is an $I$-torsion-free $R$-module. By [7, Lemma 2.1.1 (ii)], the ideal $I$ contains an element $r$ which is a non-zero divisor on $M$. The exact sequence $0 \rightarrow M \stackrel{r}{\rightarrow} M \rightarrow$ $(M / r M) \rightarrow 0$ induces an exact sequence

$$
H_{I}^{n-1}(M) \rightarrow H_{I}^{n-1}\left(\frac{M}{r M}\right) \stackrel{f}{\rightarrow} H_{I}^{n}(M) \stackrel{r}{\rightarrow} H_{l}^{n}(M) .
$$

Now we conclude from the exact sequence $0 \rightarrow \operatorname{Im} f \rightarrow H_{l}^{n}(M) \stackrel{r}{\rightarrow} H_{I}^{n}(M)$ and the fact that $r \in I$ that it suffices for us to show that $\operatorname{Hom}_{R}(R / I, \operatorname{Im} f)$ is finitely generated. Since $H_{l}^{n-1}(M)$ is finitely generated, so is $\operatorname{Ker} f$. Hence consider the exact sequence

$$
0 \rightarrow \operatorname{Ker} f \rightarrow H_{I}^{n-1}\left(\frac{M}{r M}\right) \rightarrow \operatorname{Im} f \rightarrow 0
$$

to deduce that $\operatorname{Hom}_{R}(R / I, \operatorname{Im} f)$ is finitely generated, because

$$
\operatorname{Hom}_{R}\left(R / I, H_{I}^{n-1}(M / r M)\right)
$$

is finitely generated by induction hypothesis. This completes the inductive step and so the proof.

REMARK 4.2. Let $\Phi$ be a system of ideals of $R$ and $M$ be a finitely generated $R$-module. Let $n$ be a non-negative integer such that $H_{\Phi}^{i}(M)$ is finitely generated for all $i<n$. Then $\operatorname{Hom}_{R}\left(R / I, H_{\Phi}^{n}(M)\right)$ is finitely generated for all $I \in \Phi$.

LEMMA 4.3. Let the notation be as in Remark 4.2. Then $H_{\Phi}^{i}(M) \cong \lim _{\overrightarrow{\mathfrak{a} \in \Phi}} H_{\mathfrak{a}}^{i}(M)$ for all $i \geq 0$.

ProOF. See [4, Lemma 2.1]. 
LEMMA 4.4. Let the notation be as in Remark 4.2. If $E$ is an injective $R$-module, then so is $\Gamma_{\Phi}(E)$.

ProOF. By Lemma 4.3, $\Gamma_{\Phi}(M) \cong \lim _{\vec{a} \in \Phi} \Gamma_{a}(M)$. On the other hand, for an ideal $\mathfrak{a}$ of $R$, by [7, Proposition 2.1.4], $\Gamma_{\mathrm{a}}(E)$ is injective since $R$ is Noetherian. Therefore, by $[24$, Theorem 4.10$], \Gamma_{\Phi}(E)$ is injective.

DEFINITION 4.5. We say that an $R$-module $M$ is $\Phi$-torsion if $\Gamma_{\Phi}(M)=M$. We say that $M$ is $\Phi$-torsion-free if $\Gamma_{\Phi}(M)=0$.

LEMMA 4.6. If $N$ is an $\Phi$-torsion $R$-module, then $H_{\Phi}^{i}(N)=0$ for all $i>0$.

Proof. By using Lemma 4.4, we can deduce, as in the proof of [7, Corollary 2.1.6], that for every $\Phi$-torsion $R$-module $N$, there exists an injective resolution in which each term is an $\Phi$-torsion $R$-module. Hence $H_{\Phi}^{i}(N)=0$ for all $i>0$.

COROLlaRY 4.7 (See [19, Theorem B $(\beta)$ ] and [6, Theorem 2.2]). Let I be an ideal of $R$ and $n$ be a non-negative integer such that $H_{l}^{i}(M)$ is finitely generated for all $i<n$. Then the set $\operatorname{Ass}_{R}\left(H_{I}^{n}(M)\right)$ is finite.

PROOF. It follows from Theorem 4.1 and the fact that

$$
\operatorname{Ass}_{R}\left(H_{l}^{n}(M)\right)=\operatorname{Ass}_{R}\left(\operatorname{Hom}_{R}\left(R / I, H_{l}^{n}(M)\right)\right) .
$$

The following theorem is, in fact, a general version of Theorem 3.6 in the context of general local cohomology modules. With it, we are able to prove $I$-cofiniteness in several cases.

THEOREM 4.8. Let $\Phi$ be a system of ideals of $R$ and $M$ be a finitely generated $R$-module. Suppose that $n \geq 0$ is such that $H_{\Phi}^{j}(M)$ is finitely generated for all $j<n$ and that $H_{J}^{j}(M)=0$ for all $j>n$ and all $J \in \Phi$. Then, $H_{\Phi}^{i}(M)$ is $\Phi$-cofinite for all $i$.

REMARK 4.9. Let $I$ be in $\Phi$, and put $\Phi_{I}=\{J \in \Phi \mid J \subseteq \mathfrak{a} I$ for some $\mathfrak{a} \in \Phi\}$. Then $\Phi_{l}$ is cofinal in $\Phi$. Note that $J \subseteq I$ for all $J \in \Phi_{l}$.

PROOF OF THEOREM 4.8. We have only to prove the theorem in the case $i=n$. By Proposition 2.5 and Remark 2.4, there exists an ideal $l$ in $\Phi$ such that, for all $J \in \Phi$, every $J$-filter regular $M$-sequence, of length $n$, is $l$-filter regular $M$-sequence. Now, by Remark 4.9, we may assume that $J \subseteq I$, for all $J \in \Phi$. Hence every $J$-filter regular $M$-sequence, of length $n$, is also $I$-filter regular $M$-sequence. Fix $J \in \Phi$. Let 
$x_{1}, \ldots, x_{n}$ be an $J$-filter regular $M$-sequence. Hence by Proposition 2.2 , we conclude that

$$
H_{I}^{n}(M) \cong H_{l}^{0}\left(H_{\left(x_{1}, \ldots, x_{n}\right)}^{n}(M)\right) \cong H_{I}^{0}\left(H_{J}^{0}\left(H_{\left(x_{1}, \ldots, x_{n}\right)}^{n}(M)\right)\right) \cong H_{I}^{0}\left(H_{J}^{n}(M)\right) .
$$

Since the local cohomology functor $H_{l}^{0}(-)$ commutes with direct limits, it follows that $H_{I}^{n}(M) \cong H_{I}^{0}\left(H_{\Phi}^{n}(M)\right)$. Now, by using Claim 4.10 and [7, Theorem 2.2.4], we have the exact sequence

$$
0 \rightarrow H_{l}^{n}(M) \rightarrow H_{\Phi}^{n}(M) \rightarrow D_{I}\left(H_{\Phi}^{n}(M)\right) \rightarrow 0 .
$$

Therefore we have the following long exact sequence

$$
\begin{aligned}
\cdots & \rightarrow \operatorname{Ext}_{R}^{i}\left(R / I, H_{I}^{n}(M)\right) \rightarrow \operatorname{Ext}_{R}^{i}\left(R / I, H_{\Phi}^{n}(M)\right) \\
& \rightarrow \operatorname{Ext}_{R}^{i}\left(R / I, D_{I}\left(H_{\Phi}^{n}(M)\right)\right) \rightarrow \cdots .
\end{aligned}
$$

Now, by Claim 4.11, in order to conclude the result it suffices for us to prove that $\operatorname{Ext}_{R}^{i}\left(R / I, H_{I}^{n}(M)\right)$ is finitely generated for all $i$. Note that, by Proposition 2.5 (3) and [18, Theorem (3)], $H_{i}^{i}(M)$ is finitely generated for all $i<n$. Hence the conclusion follows immediately from Theorem 3.6.

CLAIM 4.10. Let $\Phi, M, I$ and $n$ be as in Theorem 4.8. Then $H_{I}^{i}\left(H_{\Phi}^{n}(M)\right)=0$ for all $i>0$.

PROOF. Fix $J \in \Phi$. Let $x_{1}, \ldots, x_{n}$ be an $J$-filter and so $I$-filter regular $M$-sequence. Set $N:=H_{\left(x_{1}, \ldots, x_{n}\right)}^{n}(M)$. Let $E^{\bullet}$ be an injective resolution of $N$. Thus, by Lemma 4.4, $H_{J}^{0}\left(E^{\bullet}\right)$ is an injective complex. Since $H_{J}^{j}(N) \cong H_{J}^{j+n}(M)=0$ for all $j \geq 1$ and $H_{J}^{0}(-)$ is a left exact functor, $H_{J}^{0}\left(E^{\bullet}\right)$ is an injective resolution of $H_{J}^{0}(N)$. Now, it follows from Proposition 2.2 that

$$
H_{I}^{i}\left(H_{J}^{n}(M)\right) \cong H_{l}^{i}\left(H_{J}^{0}(N)\right) \cong H^{i}\left(H_{I}^{0}\left(H_{J}^{0}\left(E^{\bullet}\right)\right)\right) .
$$

Since $J \subseteq I, H_{I}^{0}\left(H_{J}^{0}\left(E^{\bullet}\right)\right) \cong H_{I}^{0}\left(E^{\bullet}\right)$ and hence

$$
H_{I}^{i}\left(H_{J}^{n}(M)\right) \cong H^{i}\left(H_{l}^{0}\left(E^{\bullet}\right)\right) \cong H_{I}^{i}(N) \cong H_{I}^{i+n}(M),
$$

where, by our assumption, the latter module is zero for all $i>0$. Now the claim follows, since $H_{I}^{0}(-)$ commutes with direct limits.

Claim 4.11. Let $\Phi, M, I$ and $n$ be as in Theorem 4.8. Then

$$
\operatorname{Ext}_{R}^{i}\left(R / I, D_{l}\left(H_{\Phi}^{n}(M)\right)\right)=0
$$

for all $i \geq 0$. 
ProOf. Let $L^{\bullet}$ be an injective resolution of $H_{\Phi}^{n}(M)$. Application of the functor $D_{I}(-)$ yields an injective complex, whose terms are all $I$-torsion-free. Observe that, as we mentioned in the proof of Claim 3.7, for an injective $R$-module $E$, $D_{I}(E)$ is injective and $I$-torsion-free. By [7, Theorem 2.2.4 (ii)], for all $i \geq 1$, $\mathscr{R}^{i} D_{l}\left(H_{\Phi}^{n}(M)\right) \cong H_{I}^{i+1}\left(H_{\Phi}^{n}(M)\right)$. Since, by the above claim, $H_{I}^{i+1}\left(H_{\Phi}^{n}(M)\right)=0$ and $D_{I}(-)$ is a left exact functor, $D_{I}\left(L^{\bullet}\right)$ is, in fact, an injective resolution of $D_{I}\left(H_{\Phi}^{n}(M)\right)$. Therefore $\operatorname{Ext}_{R}^{i}\left(R / I, D_{I}\left(H_{\Phi}^{n}(M)\right)\right)=0$ for all $i \geq 0$.

The following definitions are recoded for another result of this paper. These definitions are generalizations of the familiar concepts, 'arithmetic rank of $I$ ', denoted by ara $(I)$, and 'finiteness dimension of $M$ relative to $I$ ', denoted by $f_{I}(M)$ (see [7, Definition 3.3.2 and Definition 9.1.3]).

DEFINITION 4.12. Let $\Phi$ be a system of ideals of $R$. The arithmetic rank of $\Phi$, denoted by $\operatorname{ara}(\Phi)$, is defined as follows $\operatorname{ara}(\Phi)=\operatorname{Max}\{\operatorname{ara}(I): l \in \Phi\}$.

Note that $\operatorname{ara}(\Phi)$ is either a positive integer, whenever $\Phi$ is non-empty, or $\infty$. In particular, if $\Phi=\left\{I^{i}: i>0\right\}$, then $\operatorname{ara}(\Phi)=\operatorname{ara}(I)$.

DEFINITION 4.13 (See [1, Definition 5]). Let $M$ be a finitely generated $R$-module. We define the finiteness dimension $f_{\Phi}(M)$ of $M$ relative to $\Phi$ by

$$
\begin{aligned}
f_{\Phi}(M) & =\inf \left\{i \geq 0: H_{\Phi}^{i}(M) \text { is not finitely generated }\right\} \\
& =\inf \left\{i \geq 0: I H_{\Phi}^{i}(M) \neq 0 \text { for all } I \in \Phi\right\} .
\end{aligned}
$$

COROLLARY 4.14. Let $\Phi$ be a system of ideals of $R$. Let $M$ be a finitely generated $R$-module and $I$ be an arbitrary ideal of $R$.

(i) If $\operatorname{ara}(\Phi) \leq f_{\Phi}(M)$, then $H_{\Phi}^{i}(M)$ is $\Phi$-cofinite for all i.

(ii) If $H_{l}^{i}(M)=0$ for all $i>f_{l}(M)$, then $H_{l}^{i}(M)$ is $I$-cofinite for all $i$.

(iii) If $\operatorname{ara}(l) \leq f_{I}(M)$, then $H_{I}^{i}(M)$ is I-cofinite for all $i$.

Note that (iii) provides an improved form of the main result of [17].

ProOF. (i) If $f_{\Phi}(M)$ is infinite then there is nothing to do any more. Hence we may assume that $f_{\Phi}(M)$ is finite. So $f_{\Phi}(M)=\operatorname{ara}(\Phi)$, by [7, Corollary 3.3.3]. Now the result follows easily from Theorem 4.8 .

The statements (ii) and (iii) are immediate consequence of Theorem 3.6.

COROLLARY 4.15. Let $n$ be a positive integer and let

$$
\Phi=\left\{\sum_{i=1}^{n} R x_{i}: x_{1}, \ldots, x_{n} \text { is an } M \text {-regular sequence }\right\} \text {. }
$$


Then $H_{\Phi}^{j}(M)$ is $\Phi$-cofinite for all $j$.

ProOF. Note that, by [26, Example 3.10] and [5, Theorem], $\Phi$ is a system of ideals of $R$. Now use [7, Theorem 6.2.7 and Corollary 3.3.3] and then Theorem 4.8.

There are some results on the finiteness of the Bass numbers of locall cohomology modules (compare $[15,16,20,17])$. For a prime ideal $\mathfrak{p}$ of $R$ and an $R$-module $W$, the $i$-th Bass number $\mu^{i}(\mathfrak{p}, W)$, is defined to be $\operatorname{dim}_{k(\mathfrak{p})} \operatorname{Ext}_{R_{\mathfrak{p}}}^{i}\left(k(\mathfrak{p}), W_{\mathfrak{p}}\right)$, where $k(\mathfrak{p})=$ $R_{\mathfrak{p}} / \mathfrak{p} R_{\mathfrak{p}}$ (compare [2, Lemma 2.7]). If $W$ is $I$-cofinite, then, by [14, Lemma 4.2], the Bass numbers of $W$ are finite. Hence one can put all our various results together to obtain some corollaries about the finiteness of the Bass numbers of local cohomology modules.

\section{Acknowledgments}

The authors are deeply grateful to the referees for their careful reading of the manuscript and helpful suggestions. We would like to thank Professor H. Zakeri for helpful discussion, and also Institute for Studies in Theoretical Physics and Mathematics (IPM) for the financial support.

\section{References}

[1] J. Asadollahi, K. Khashyarmanesh and Sh. Salarian, 'Local-global principle for annihilation of general local cohomology', Collog. Math. 87 (2001), 129-136.

[2] H. Bass, 'On the ubiquity of Gorenstein rings', Math. Z. 82 (1963), 8-28.

[3] M. H. Bijan-Zadeh, 'Torsion theories and local cohomology over commutative Noetherian rings', J. London Math. Soc. 19 (1979), 402-410.

[4] _ 'A common generalization of local cohomology theories', Glasgow Math. J. 21 (1980), 173-181.

[5] - 'Modules of generalized fractions and general local cohomology modules', Arch. Math. 48 (1987), 58-62.

[6] M. Brodmann and A. Lashgari Faghani, 'A finiteness result for associated primes of local cohomology modules', Proc. Amer. Math. Soc. 128 (2000), 2851-2853.

[7] M. Brodmann and R.Y. Sharp, Local cohomology-an algebraic introduction with geometric applications, Cambridge Stud. Adv. Math. 60 (Cambridge University Press, Cambridge, 1998).

[8] D. Delfino, 'On the cofiniteness of local cohomology modules', Math. Proc. Cambridge Philos. Soc. 115 (1994), 79-84.

[9] D. Delfino and T. Marley, 'Cofinite modules and local cohomology', J. Pure Appl. Algebra 121 (1997), 45-52.

[10] A. Grothendieck, Local cohomology notes by R. Hartshorne, Lecture Notes in Math. 862 (Springer, New York, 1966).

[11] - Cohomologie locale des faisceaux cohérents et théorèmes de Lefshetz locaux et globaux (North Holand, 1969). 
[12] R. Hartshorne, 'Affine duality and cofiniteness', Invent. Math. 9 (1970), 145-164.

[13] C. Huneke, 'The theory of $d$-sequences and power of ideals', Adv. Math. 46 (1982), 249-279.

[14] C. Huneke and J. Koh, 'Cofiniteness and vanishing of local cohomology modules', Math. Proc. Cambridge Philos. Soc. 110 (1991), 421-429.

[15] C. Huneke and R. Y. Sharp, 'Bass numbers of local cohomology modules', Trans. Amer. Math. Soc. 339 (1993), 765-779.

[16] K.-I. Kawasaki, 'On the finiteness of bass numbers of local cohomology modules', Proc. Amer. Math. Soc. 124 (1996), 3275-3279.

[17] - 'Cofiniteness of local cohomology modules for principal ideals', Bull. London Math. Soc. 30 (1998), 241-246.

[18] K. Khashyarmanesh and Sh. Salarian, 'Filter regular sequences and the finiteness of local cohomology modules', Comm. Algebra (8) 26 (1998), 2483-2490.

[19] $\longrightarrow$, 'On the associated primes of local cohomology modules', Comm. Algebra (12) 27 (1999), 6191-6198.

[20] G. Lyubeznik, 'Finiteness properties of local cohomology modules (an application of $D$-modules to commutative algebra)', Invent. Math. 102 (1993), 41-55.

[21] E. Matlis, 'Injective modules over noetherian rings', Pacific J. Math. 8 (1958), 511-528.

[22] U. Nagel and P. Schenzel, 'Cohomological annihilators and Castelnuovo-Mumford regularity', in: Commutative algebra: Syzygies, multiplicities, and birational algebra (South Hadley, MA, 1992), Contemp. Math. 159 (Amer. Math. Soc., Providence, RI, 1994) pp. 307-328.

[23] C. Peskine and L. Szpiro, 'Dimension projective finie et cohomologie locale', I.H.E.S. 42 (1973), 323-395.

[24] J. Rotman, An introduction to homological algebra (Academic Press, Orlando, FL, 1979).

[25] P. Schenzel, N. V. Trung and N. T. Cuong, 'Verallgemeinerte Cohen-Macaulay-Moduln', Math. Nachr. 85 (1978), 57-73.

[26] R. Y. Sharp and H. Zakeri, 'Modules of generalized fractions', Mathematika 29 (1982), 32-41.

[27] J. Stuckrad and W. Vogel, Buchsbaum rings and applications (VEB Deutscher Verlag der Wissenschaften, Berlin, 1986).

[28] N. V. Trung, 'Absolutely superficial sequences', Math. Proc. Cambridge Philos. Soc. 93 (1983), $35-47$.

[29] K.-I. Yoshida, 'Cofiniteness of local cohomology modules for ideals of dimension one', Nagoya Math. J. 147 (1997), 179-191.

Institute for Studies in Theoretical

Physics and Mathematics

P.O. Box 19395-5746, Tehran

Iran

and

Shahre-e-Kord University

Faculty of Science

P.O.Box 115, Shahre-e-Kord

Iran

e-mail: Asadollahi@mail.ipm.ir
Institute for Studies in Theoretical Physics and Mathematics P.O. Box 19395-5746, Tehran Iran and

Damghan University Department of Mathematics P.O. Box 36715-364, Damghan Iran e-mail: Khashyar@mail.ipm.ir Salarian@mail.ipm.ir 